\title{
Obesidade, Sobrepeso, Adiposidade Corporal e Risco Cardiovascular em Crianças e Adolescentes
}

\author{
Obesity, Overweight, Body Adiposity and Cardiovascular Risk in Children and Adolescents
}

\author{
Weimar Kunz Sebba Barroso ${ }^{1 \oplus}$ e Ana Luiza Lima Souza ${ }^{1,2}$ \\ Universidade Federal de Goiás - Liga de Hipertensão Arterial, ${ }^{1}$ Goiânia, GO - Brasil \\ Universidade Federal de Goiás - Programa de Pós-Graduação em Ciências da Saúde, ${ }^{2}$ Goiânia, GO - Brasil \\ Minieditorial referente ao artigo: Adiposidade Corporal e Apolipoproteínas em Crianças e Adolescentes: Metanálise de Estudos Prospectivos
}

A obesidade e o sobrepeso são considerados um problema de saúde pública global e que contribuem fortemente para várias doenças crônicas não transmissíveis (DCNT), dentre elas a síndrome metabólica, diabetes mellitus (DM), doenças cardiovasculares (DCV) e câncer. São estimados mais de 1.9 bilhões de adultos com sobrepeso, o que representa 39\% da população mundial, e 13\% de adultos obesos. A Organização Mundial da Saúde estimou, para 2019, mais de 38 milhões de crianças abaixo dos cinco anos com sobrepeso ou obesidade. A obesidade na infância está associada a maior chance de morte prematura, aumento do risco de hipertensão arterial, DM e câncer. Além disso, crianças obesas apresentam marcadores precoces de DCV, aumento do risco de fraturas, dificuldades respiratórias e resistência à insulina. ${ }^{1}$

A obesidade, como fator de risco independente para doenças cardiovasculares, ${ }^{2}$ está relacionada com a elevação dos níveis de apolipoproteínas B (ApoB) e consequente disfunção endotelial. A presença de obesidade e dislipidemias durante a infância reflete o desenvolvimento de morbidades cardiovasculares na idade adulta. ${ }^{3}$

O excesso de adiposidade corporal está relacionado com a presença de dislipidemia, identificada a partir do aumento dos níveis de colesterol total sérico e lipoproteínas de baixa e alta densidade. Ainda, entendendo que a dislipidemia aterogênica e doença aterosclerótica podem ter o seu início na infância e podem estar acompanhadas da obesidade, elas deve ser analisadas como fatores de risco associados à presença da doença coronária (DAC) na fase adulta. ${ }^{2,4-6}$

Altas concentrações de ApoB e baixas concentrações de ApoA1 têm sido identificadas como marcadores bioquímicos para aterosclerose mesmo em idades mais precoces, ${ }^{7}$ estando associadas com circunferência da cintura, adiposidade e história familiar de DAC. ${ }^{8}$

\section{Palavras-chave}

Criança; Adolescente; Hipertensão; Diabetes Mellitus; Síndrome Metabólica; Sobrepeso; Obesidade; Fatores de Risco; Saúde Pública.

Correspondência: Weimar Kunz Sebba Barroso•

Universidade Federal de Goiás - Liga de Hipertensão Arterial - Av.

Universitária Hospital Das Clínicas. CEP 74605-220, Goiânia, GO - Brasil

E-mail: sebbabarroso@gmail.com

DOI: https://doi.org/10.36660/abc.20200540
As apolipoproteínas A1 e B são proteínas essenciais para o metabolismo das partículas de lipoproteínas e seus níveis séricos são reconhecidos como preditores de risco para doença aterosclerótica. A avaliação dos níveis plasmáticos pode auxiliar na identificação do aumento do risco e adoção de estratégias de intervenção precoce. Têm, portanto, a capacidade de adicionar informações clínicas que vão além daquelas obtidas pela avaliação do LDL e HDL. 9,10

Em adultos, as altas taxas de ApoB estão associadas fortemente com a síndrome metabólica e obesidade e são melhores preditoras de risco cardiovascular que as medidas tradicionais de lipídeos sanguíneos. Na população jovem, o perfil lipídico convencional não é um bom preditor de DAC na idade adulta. , $10-12^{2}$

Na revisão sistemática "Adiposidade corporal e apolipoproteínas em crianças e adolescentes: metanálise de estudos prospectivos", ${ }^{13}$ a ApoB foi registrada como um marcador cardiometabólico associado com a massa corporal entre adolescentes e crianças, indicando alteração no perfil das apolipoproteínas nessa população.

A relevância desse estudo ${ }^{13}$ pode ser destacada não só pelo achado clínico, definindo relações entre morbidades e biomarcadores, mas também pelo fato de ter sido direcionado para a população de crianças e adolescentes. Os resultados sensibilizam para a necessidade de estratégias de enfrentamento coletivo para problemas de magnitude global como a obesidade e as doenças cardiovasculares.

A inclusão das apolipoproteínas na avaliação padrão do perfil lipídico, como biomarcadores sensíveis para a identificação de risco, pode ser útil como estratégia de rastreamento e detecção precoce, além da construção de indicadores para a vigilância da saúde nessa população. 


\section{Referências}

1. World Health Organization.(WHO) Obesity and overweight [Internet]. Geneva: WHO; 2018 [acesso em 26 maio 2020]. Disponível em: http:// www.who.int/en/news-room/fact-sheets/detail/obesity-and-overweight.

2. Ades PA,Savage PD. Obesity in coronary heart disease: an unaddressed behavioral risk factor. Prev Med. 2017 Nov;104:117-9.

3. Palmeira AC, Leal AA, Ramos NMN, Neto JAF, Simões MOS, Medeiros CCM. Lipoprotein (a) and cardiovascular risk factors in children and adolescents. Rev Paul Pediatr. 2013;31(4):531-7.

4. Montazerifar F, Bolouri A, Mahmoudi Mozaffar M, Karajibani M. The prevalence of metabolic syndrome in coronary artery disease patients. Cardiol Res. 2016;7(6):202-8.

5. Dhungana SP, Mahato AK, Ghimire R, Shreewastav RK. Prevalence of dyslipidemia in patients with acute coronary syndrome admitted at Tertiary Care Hospital in Nepal: a descriptive cross-sectional study. JNMA J Nepal Med Assoc. 2020;58(224):204-8.

6. Lee $\mathrm{YH}$, Choi SH, Lee KW, Kim DJ. Apolipoprotein B/A1 ratio is associated with free androgen index and visceral adiposity and may be an indicator of metabolic syndrome in male children and adolescents. Clin Endocrinol (Oxf). 2011;74(5):579-86.

7. Aditya GP, Bari MS, Bari MA, Mutalib MA, Paul GK. Association of metabolic syndrome with hyper apolipoprotein B status in young people with acute coronary syndrome. Mymensingh Med J. 2017;26(1):68-74.
8. Khalil A, Aggarwal A, Arora S, Bhattacharya J. Lipoprotein (a)--lipid profile and apolipoprotein B in children of young parents with coronary artery disease. Indian Heart J. 2011;63(5):450-3.

9. Aditya GP, Bari MA. Apolipoprotein B versus non- high density lipoprotein cholesterol as a discriminating factor for acute coronary syndrome in young people. Mymensingh Med J. 2016;25(3):458-64.

10. Bari MA, Aditya GP, Bhuiyan AS, Ahmed MU, Islam MZ, Rahman MM, et al. Measurement of apolipoprotein B may predict acute coronary syndrome in hyper-triglyceridemic young population. Mymensingh Med J. $2015 ; 24(2): 257-62$

11. Soria-Florido MT, Castaner O, Lassale C, Estruch R, Salas-Salvadó J, Martínez Gonzáles MA, et al. Dysfunctional high-density lipoproteins are associated with a greater incidence of acute coronary syndrome in a population at high cardiovascular risk: a nested case-control study. Circulation. 2020;141(6):444-53.

12. Aditya GP, Bari MS, Bari MA, Mutalib MA, Islam MZ, Paul GK, et al. Risk of acute coronary syndrome is better predicted by apolipoprotein $B$ in young people than dyslipidemic parameter of conventional lipid profile. Mymensingh Med J. 2016;25(4):663-8.

13. Jesus GS, Costa PRF, Oliveira LPM, Queiroz VAO, Cunha CM, Pereira EM et al. Body Adiposity and Apolipoproteins in Children and Adolescents: A Meta-Analysis of Prospective Studies. Arq Bras Cardiol. 2020 115(2):163-171. 